Nanhua Xi

\title{
Kazhdan-Lusztig basis and a geometric filtration of an affine Hecke algebra, II
}

\author{
Received February 15, 2008 and in revised form November 25, 2008
}

\begin{abstract}
An affine Hecke algebra can be realized as an equivariant K-group of the corresponding Steinberg variety. This gives rise naturally to some two-sided ideals of the affine Hecke algebra by means of the closures of nilpotent orbits of the corresponding Lie algebra. In this paper we will show that the two-sided ideals are in fact the two-sided ideals of the affine Hecke algebra defined through the two-sided cells of the corresponding affine Weyl group after the two kinds of ideals are tensored by $\mathbb{Q}$. This proves a weak form of a conjecture of Ginzburg proposed in 1987.
\end{abstract}

\section{Introduction}

Let $H$ be an affine Hecke algebra over the ring $\mathbb{Z}\left[v, v^{-1}\right]$ of Laurent polynomials in an indeterminate $v$ with integer coefficients. The affine Hecke algebra has a Kazhdan-Lusztig basis. The basis has many remarkable properties and plays an important role in representation theory. Also, Kazhdan and Lusztig and Ginzburg gave a geometric realization of $H$, which is the key to the proof by Kazhdan and Lusztig of the Deligne-Langlands conjecture on classification of irreducible modules of affine Hecke algebras over $\mathbb{C}$ at non-roots of 1 . This geometric construction of $H$ has some two-sided ideals defined naturally by means of the nilpotent variety of the corresponding Lie algebra. The two-sided ideals form a nice filtration of the affine Hecke algebra. In [G2] Ginzburg conjectured that the two-sided ideals are in fact the two-sided ideals of the affine Hecke algebra defined through two-sided cells of the corresponding affine Weyl group (see also [L6, T2]). The conjecture is known to be true for the trivial nilpotent orbit $\{0\}$ (see Corollary 8.13 in [L6] and Theorem 7.4 in [X1]) and for type A [TX]. Other evidence is showed in [L6] Corollary 9.13]. We will prove the two kinds of two-sided ideals coincide after they are tensored by $\mathbb{Q}$ (see Theorem 1.5 in Section 1). This proves a weak form of Ginzburg's conjecture.

\section{Affine Hecke algebra}

1.1. Let $G$ be a simply connected simple algebraic group over the complex number field $\mathbb{C}$. The Weyl group $W_{0}$ acts naturally on the character group $X$ of a maximal tours

N. Xi: Institute of Mathematics, Chinese Academy of Sciences, Beijing, 100080, China;

e-mail: nanhua@math.ac.cn

Mathematics Subject Classification (2010): Primary 20C08 
of $G$. The semidirect product $W=W_{0} \ltimes X$ with respect to this action is called an (extended) affine Weyl group. Let $H$ be the associated Hecke algebra over the ring $\mathcal{A}=$ $\mathbb{Z}\left[v, v^{-1}\right]$ ( $v$ an indeterminate) with parameter $v^{2}$. Thus $H$ has an $\mathcal{A}$-basis $\left\{T_{w} \mid w \in W\right\}$ and its multiplication is defined by the relations $\left(T_{s}-v^{2}\right)\left(T_{s}+1\right)=0$ if $s$ is a simple reflection and $T_{w} T_{u}=T_{w u}$ if $l(w u)=l(w)+l(u)$, where $l$ is the length function of $W$.

1.2. Let $\mathfrak{g}$ be the Lie algebra of $G, \mathcal{N}$ the nilpotent cone of $\mathfrak{g}$, and $\mathcal{B}$ the variety of all Borel subalgebras of $\mathfrak{g}$. The Steinberg variety $Z$ is the subvariety of $\mathcal{N} \times \mathcal{B} \times \mathcal{B}$ consisting of all triples $\left(n, \mathfrak{b}, \mathfrak{b}^{\prime}\right), n \in \mathfrak{b} \cap \mathfrak{b}^{\prime} \cap \mathcal{N}, \mathfrak{b}, \mathfrak{b}^{\prime} \in \mathcal{B}$. Let $\Lambda=\{(n, \mathfrak{b}) \mid n \in \mathcal{N} \cap \mathfrak{b}, \mathfrak{b} \in \mathcal{B}\}$ be the cotangent bundle of $\mathcal{B}$. Clearly $Z$ can be regarded as a subvariety of $\Lambda \times \Lambda$ via the imbedding $Z \rightarrow \Lambda \times \Lambda,\left(n, \mathfrak{b}, \mathfrak{b}^{\prime}\right) \mapsto\left(n, \mathfrak{b}, n, \mathfrak{b}^{\prime}\right)$. Define a $G \times \mathbb{C}^{*}$-action on $\Lambda$ by $(g, z):(n, \mathfrak{b}) \mapsto\left(z^{-2} \operatorname{ad}(g) n, \operatorname{ad}(g) \mathfrak{b}\right)$. Let $G \times \mathbb{C}^{*}$ act on $\Lambda \times \Lambda$ diagonally; then $Z$ is a $G \times \mathbb{C}^{*}$-stable subvariety of $\Lambda \times \Lambda$. For $1 \leq i<j \leq 3$, let $p_{i j}$ be the projection from $\Lambda \times \Lambda \times \Lambda$ to its $(i, j)$-factor. Note that the restriction of $p_{13}$ gives rise to a proper morphism $p_{12}^{-1}(Z) \cap p_{23}^{-1}(Z) \rightarrow Z$. Let $K^{G \times \mathbb{C}^{*}}(Z)=K^{G \times \mathbb{C}^{*}}(\Lambda \times \Lambda ; Z)$ be the Grothendieck group of the category of $G \times \mathbb{C}^{*}$-equivariant coherent sheaves on $\Lambda \times \Lambda$ with support in $Z$. We define the convolution product

$*: K^{G \times \mathbb{C}^{*}}(Z) \times K^{G \times \mathbb{C}^{*}}(Z) \rightarrow K^{G \times \mathbb{C}^{*}}(Z), \quad \mathscr{F} * \mathscr{G}=\left(p_{13}\right)_{*}\left(p_{12}^{*} \mathscr{F} \otimes_{\mathcal{O}_{\Lambda \times \Lambda \times \Lambda}} p_{23}^{*} \mathscr{G}\right)$,

where $\mathcal{O}_{\Lambda \times \Lambda \times \Lambda}$ is the structure sheaf of $\Lambda \times \Lambda \times \Lambda$. This endows $K^{G \times \mathbb{C}^{*}}(Z)$ with an associative algebra structure over the representation ring $R_{G \times \mathbb{C}^{*}}$ of $G \times \mathbb{C}^{*}$. We shall regard the indeterminate $v$ as the representation $G \times \mathbb{C}^{*} \rightarrow \mathbb{C}^{*},(g, z) \mapsto z$. Then $R_{G \times \mathbb{C}^{*}}$ is identified with $\mathcal{A} \otimes_{\mathbb{Z}} R_{G}$. In particular, $K^{G \times \mathbb{C}^{*}}(Z)$ is an $\mathcal{A}$-algebra. Moreover, as an $\mathcal{A}$-algebra, $K^{G \times \mathbb{C}^{*}}(Z)$ is isomorphic to the Hecke algebra $H$ (see [G1, KL2] or [CG] L6]). We shall identify $K^{G \times \mathbb{C}^{*}}(Z)$ with $H$.

1.3. Let $\mathcal{C}$ and $\mathcal{C}^{\prime}$ be two $G$-orbits in $\mathcal{N}$. We say that $\mathcal{C} \leq \mathcal{C}^{\prime}$ if $\mathcal{C}$ is in the closure of $\mathcal{C}^{\prime}$. This defines a partial order on the set of $G$-orbits in $\mathcal{N}$. Given a locally closed $G$-stable subvariety of $\mathcal{N}$, we set $Z_{Y}=\left\{\left(n, \mathfrak{b}, \mathfrak{b}^{\prime}\right) \in Z \mid n \in Y\right\}$.

If $Y$ is closed, then the inclusion $i_{Y}: Z_{Y} \rightarrow Z$ induces a map $\left(i_{Y}\right)_{*}: K^{G \times \mathbb{C}^{*}}\left(Z_{Y}\right) \rightarrow$ $K^{G \times \mathbb{C}^{*}}(Z)$ (see [G1, KL2]). The image $H_{Y}$ of $\left(i_{Y}\right)_{*}$ is in fact a two-sided ideal of $K^{G \times \mathbb{C}^{*}}(Z)$ (see [L6, Corollary 9.13]), which is generated by $G \times \mathbb{C}^{*}$-equivariant sheaves supported on $Z_{Y}$. It is conjectured that this ideal is spanned by elements in a KazhdanLusztig basis (see [G2, L6, T2]).

1.4. Let $C_{w}=v^{-l(w)} \sum_{y \leq w} P_{y, w}\left(v^{2}\right) T_{y}$, where $P_{y, w}$ are the Kazhdan-Lusztig polynomials. Then the elements $\bar{C}_{w}(w \in W)$ form an $\mathcal{A}$-basis of $H$, called a Kazhdan-Lusztig basis of $H$. Define $w \leq_{L R} u$ if $a_{w} \neq 0$ in the expression $h C_{u} h^{\prime}=\sum_{z \in W} a_{z} C_{z}\left(a_{z} \in \mathcal{A}\right)$ for some $h, h^{\prime}$ in $H$. This defines a preorder on $W$. The corresponding equivalence classes are called two-sided cells and the preorder gives rise to a partial order $\leq_{L R}$ on the set of two-sided cells of $W$. (See [KL1].) For an element $w$ in $W$ and a two-sided cell $c$ of $W$ we shall write $w \leq_{L R} c$ if $w \leq_{L R} u$ for some (equivalent any) $u$ in $c$.

Lusztig established a bijection between the set of $G$-orbits in $\mathcal{N}$ and the set of twosided cells of $W$ (see [L4, Theorem 4.8]). Lusztig's bijection preserves the partial orders we have defined: this was conjectured by Lusztig and verified by Bezrukavnikov (see [B, 
Theorem 4(b)]). Perhaps this bijection is at the heart of the theory of cells in affine Weyl groups; many deep results are related to it. Now we can state the main result of this paper.

Theorem 1.5. Let $\mathcal{C}$ be a $G$-orbit in $\mathcal{N}$ and $c$ the two-sided cell of $W$ corresponding to $\mathcal{C}$ under Lusztig's bijection. Then the elements $C_{w}\left(w \leq_{L R} \quad c\right)$ form a $\mathbb{Q}\left[v, v^{-1}\right]$ basis of $H_{\overline{\mathcal{C}}} \otimes_{\mathbb{Z}} \mathbb{Q}$, where $\overline{\mathcal{C}}$ denotes the closure of $\mathcal{C}$ and $\bar{H}_{\overline{\mathcal{C}}}$ is the image of the map $\left(i_{\overline{\mathcal{C}}}\right)_{*}: K^{G \times \mathbb{C}^{*}}\left(Z_{\overline{\mathcal{C}}}\right) \rightarrow K^{G \times \mathbb{C}^{*}}(Z)=H$.

Remark. In [B] Bezrukavnikov established a closely related result, which involves affine flag manifolds, derived categories and the Springer resolution (see Theorem 4(a) there). Bezrukavnikov's result deals with canonical left cells and suggests a very nice possible approach to Theorem 1.5. We will discuss this approach in Section 3. I am very grateful to the referee for pointing out this approach.

\section{Proof of the theorem}

2.1. Before proving the theorem we need to recall some results about representations of an affine Hecke algebra. Let $\mathbf{H}=\mathbb{C}\left[v, v^{-1}\right] \otimes_{\mathcal{A}} H$ and for any nonzero complex number $q$ set $\mathbf{H}_{q}=\mathbf{H} \otimes_{\mathbb{C}\left[v, v^{-1}\right]} \mathbb{C}$, where $\mathbb{C}$ is regarded as a $\mathbb{C}\left[v, v^{-1}\right]$-algebra by specializing $v$ to a square root of $q$.

For any $G$-stable locally closed subvariety $Y$ of $\mathcal{N}$ we set $\mathbf{K}^{G \times \mathbb{C}^{*}}\left(Z_{Y}\right)=$ $K^{G \times \mathbb{C}^{*}}\left(Z_{Y}\right) \otimes \mathbb{C}$. If $Y$ is closed, then the inclusion $i_{Y}: Z_{Y} \rightarrow Z$ induces an injective map $\left(i_{Y}\right)_{*}: \mathbf{K}^{G \times \mathbb{C}^{*}}\left(Z_{Y}\right) \hookrightarrow \mathbf{K}^{G \times \mathbb{C}^{*}}(Z)=\mathbf{H}$. If $Y$ is a closed subset of $\mathcal{N}$, we shall identify $\mathbf{K}^{G \times \mathbb{C}^{*}}\left(Z_{Y}\right)$ with the image of $\left(i_{Y}\right)_{*}$, which is a two-sided ideal of $\mathbf{H}$. See [KL2, 5.3] or [L6, Corollary 9.13].

Let $s$ be a semisimple element of $G$, and $n$ a nilpotent element in $\mathcal{N}$ such that $\operatorname{ad}(s) n=$ $q n$, where $q$ is in $\mathbb{C}^{*}$. Let $\mathcal{B}_{n}^{s}$ be the subvariety of $\mathcal{B}$ consisting of the Borel subalgebras containing $n$ and fixed by $s$. Then the component group $A(s, n)=C_{G}(s, n) / C_{G}(s, n)^{o}$ of the simultaneous centralizer in $G$ of $s$ and $n$ acts on the total complex homology group $H_{*}\left(\mathcal{B}_{n}^{s}\right)$. Let $\rho$ be a representation of $A(s, n)$ appearing in the space $H_{*}\left(\mathcal{B}_{n}^{s}\right)$. It is known that if $\sum_{w \in W_{0}} q^{l(w)} \neq 0$ then the isomorphism classes of irreducible representations of $\mathbf{H}_{q}$ are in one-to-one correspondence to the $G$-conjugacy classes of all the triples $(s, n, \rho)$, where $s \in G$ is semisimple, $n \in \mathcal{N}$ satisfies ad $(s) n=q n$, and $\rho$ is an irreducible representation of $A(s, n)$ appearing in $H_{*}\left(\mathcal{B}_{n}^{s}\right)$. See [KL2, X3].

Remark. In the proof of this section we shall often use arguments from [KL2] although the setting there is different from ours. In [KL2] equivariant topological K-homology $K_{\text {top }}$ ( ) is considered, while we consider equivariant algebraic K-theory $K($ ). We explain why the arguments of [KL2] work in the present paper. Besides the fact that algebraic K-theory and topological K-theory share many properties (one may compare [KL2] with $[$ [Th1, Th2, $\mathrm{CG}]$ ), the key reason is that $K\left(\mathcal{B}_{n}^{s}\right) \simeq K_{\text {top }}\left(\mathcal{B}_{n}^{s}\right)$ and $K\left(\mathcal{B}_{n}^{s}\right) \otimes \mathbb{C} \simeq K_{\text {top }}\left(\mathcal{B}_{n}^{s}\right)$ $\otimes \mathbb{C}$, as explained in [L5, p. 80]. (The isomorphisms rely on the results in [DLP].) One may see that the properties of $K_{\text {top }}\left(\mathcal{B}_{n}^{s}\right) \otimes \mathbb{C}$ play a key role in the arguments of [KL2].

2.2. From now on we assume that $q$ is not a root of 1 . Let $L_{q}(s, n, \rho)$ be an irreducible representation of $\mathbf{H}_{q}$ corresponding to the triple $(s, n, \rho)$. Kazhdan and Lusztig con- 
structed a standard module $M(s, n, q, \rho)$ over $\mathbf{H}_{q}$ such that $L_{q}(s, n, \rho)$ is the unique simple quotient of $M(s, n, q, \rho)$ (see [KL2, 5.12(b) and Theorem 7.12]). We shall write $M_{q}(s, n, \rho)$ for $M(s, n, q, \rho)$. The following simple fact will be needed.

(a) Let $\mathcal{C}$ be a $G$-orbit in $\mathcal{N}$. Then the image $H_{\overline{\mathcal{C}}}$ of $\left(i_{\overline{\mathcal{C}}}\right)_{*}$ acts on $M_{q}(s, n, \rho)$ and $L_{q}(s, n, \rho)$ by zero if $n$ is not in $\overline{\mathcal{C}}$.

Proof. Clearly $Y=\overline{\mathcal{C}} \cup(\overline{G . n}-G . n)$ is closed. If $n$ is not in $\overline{\mathcal{C}}$, then the complement in $X=\overline{\mathcal{C}} \cup \overline{G . n}$ of $Y$ is $G . n$. Recall that $\mathbf{K}^{G \times \mathbb{C}^{*}}\left(Z_{Y^{\prime}}\right)$ is regarded as a two-sided ideal of $\mathbf{H}$ for any closed subset $Y^{\prime}$ of $\mathcal{N}$ (see 2.1). According to [KL2, 5.3(c), (d) and (e)], the inclusions $i: Y \hookrightarrow X$ and $j: G . n \hookrightarrow X$ induce an exact sequence of $\mathbf{H}$-bimodules

$$
0 \rightarrow \mathbf{K}^{G \times \mathbb{C}^{*}}\left(Z_{Y}\right) \rightarrow \mathbf{K}^{G \times \mathbb{C}^{*}}\left(Z_{X}\right) \rightarrow \mathbf{K}^{G \times \mathbb{C}^{*}}\left(Z_{G . n}\right) \rightarrow 0 .
$$

Using [KL2, 5.3(e)] we know the inclusion $k: \overline{\mathcal{C}} \rightarrow Y$ induces an injective $\mathbf{H}$-bimodule homomorphism $k_{*}: \mathbf{K}^{G \times \mathbb{C}^{*}}\left(Z_{\overline{\mathcal{C}}}\right) \rightarrow \mathbf{K}^{G \times \mathbb{C}^{*}}\left(Z_{Y}\right)$. Since $M_{q}(s, n, \rho)$ is a quotient module of $\mathbf{K}^{G \times \mathbb{C}^{*}}\left(Z_{G . n}\right)$ (cf. proof of 5.13 in [KL2]), the statement (a) then follows from the exact sequence above.

2.3. Let $J_{c}$ be the based ring of a two-sided cell $c$ of $W$, which has a $\mathbb{Z}$-basis $\left\{t_{w} \mid w \in c\right\}$. Let $D_{c}$ be the set of distinguished involutions in $c$. For $x, y \in W$, we write $C_{x} C_{y}=$ $\sum_{z \in W} h_{x, y, z} C_{z}, h_{x, y, z} \in \mathcal{A}$. The map

$$
\varphi_{c}\left(C_{w}\right)=\sum_{\substack{d \in D_{c} \\ u \in W \\ a(d)=a(u)}} h_{w, d, u} t_{u}, \quad w \in W,
$$

defines an $\mathcal{A}$-algebra homomorphism $H \rightarrow J_{c} \otimes_{\mathbb{Z}} \mathcal{A}$, where $a: W \rightarrow \mathbb{N}$ is the $a$ function defined in $[\mathrm{L} 1,2.1]$. The homomorphism $\varphi_{c}$ induces a $\mathbb{C}$-algebra homomorphism $\varphi_{c, q}: \mathbf{H}_{q} \rightarrow \mathbf{J}_{c}=J_{c} \otimes_{\mathbb{Z}} \mathbb{C}$. If $E$ is a $\mathbf{J}_{c}$-module, then through $\varphi_{c, q}, E$ gets an $\mathbf{H}_{q}$-module structure, which will be denoted by $E_{q}$. See [L2, L3].

Let $\mathcal{C}$ be the nilpotent orbit corresponding to $c$. According to [L4, Theorems 4.2 and 4.8], the map $E \rightarrow E_{q}$ defines a bijection between the isomorphism classes of simple $\mathbf{J}_{c}$-modules and the isomorphism classes of standard modules $M_{q}(s, n, \rho)$ with $n$ in $\mathcal{C}$. The following fact will be needed.

(a) Let $c$ be a two-sided cell of $W$ and $\mathcal{C}$ the corresponding nilpotent class. Let $M_{q}(s, n, \rho)$ be a standard module with $n$ in a nilpotent class $\mathcal{C}^{\prime}$. If $C_{w} M_{q}(s, n, \rho) \neq 0$ for some $w \in c$, then $\overline{\mathcal{C}}^{\prime} \subseteq \overline{\mathcal{C}}$.

Proof. Let $c^{\prime}$ be the two-sided cell corresponding to $\mathcal{C}^{\prime}$. Then $M_{q}(s, n, \rho)$ is isomorphic to $E_{q}$ for some simple $\mathbf{J}_{c^{\prime}}$-module $E$. Thus $C_{w} M_{q}(s, n, \rho) \neq 0$ implies that $\varphi_{c^{\prime}, q}\left(C_{w}\right) E \neq 0$. So $h_{w, d, u} \neq 0$ for some distinguished involution $d \in c^{\prime}$ and some $u \in c^{\prime}$. We then have $c^{\prime} \leq_{L R} c$. By [B] Theorem 4(b)] we know that $\overline{\mathcal{C}}^{\prime} \subseteq \overline{\mathcal{C}}$. The statement is proved.

Now we start to prove Theorem 1.5. 
2.4. We first show that $H_{\bar{C}}$ is contained in the two-sided ideal $H^{\leq c}$ of $H$ spanned by all $C_{w}\left(w \leq_{L R} c\right)$.

Let $\mathcal{C}=G . n$ and recall that $H_{\overline{\mathcal{C}}}$ stands for the image of $\left(i_{\overline{\mathcal{C}}}\right)_{*}: K^{G \times \mathbb{C}^{*}}\left(Z_{\overline{\mathcal{C}}}\right) \rightarrow$ $K^{G \times \mathbb{C}^{*}}(Z)=H$. If $H_{\overline{\mathcal{C}}}$ were not contained in the $\mathcal{A}$-submodule $H^{\leq c}$ of $H$, we could

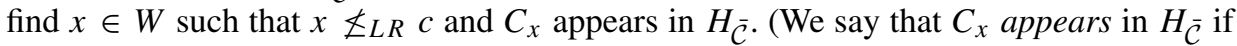
there exists an element $\sum_{w \in W} a_{w} C_{w}\left(a_{w} \in \mathcal{A}\right)$ in $H_{\overline{\mathcal{C}}}$ such that $a_{x} \neq 0$.) Choose $x \in W$ such that $C_{x}$ appears in $H_{\overline{\mathcal{C}}}, x{\mathbb{Z}_{L R}} c$ and $x$ is highest with respect to the preorder $\leq_{L R}$ and to $H_{\overline{\mathcal{C}}}$ in the following sense: whenever $C_{w}$ appears in $H_{\overline{\mathcal{C}}}$, then either $w$ and $x$ are in

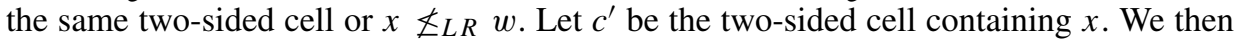
have $c^{\prime} \Varangle_{L R} c$.

Choose an element $h=\sum_{w \in W} a_{w} C_{w}\left(a_{w} \in \mathcal{A}\right)$ in $H_{\overline{\mathcal{C}}}$ such that $h_{c^{\prime}}=\sum_{w \in c^{\prime}} a_{w} C_{w}$ is nonzero. We have $\varphi_{c^{\prime}}(h)=\varphi_{c^{\prime}}\left(h_{c^{\prime}}\right)$.

We claim that $\varphi_{c^{\prime}}\left(h_{c^{\prime}}\right)$ is nonzero. Let $u \in c^{\prime}$ be such that $a_{u}$ has the highest degree (as a Laurent polynomial in $v$ ) among all $a_{w}, w \in c^{\prime}$. Let $d$ be the distinguished involution such that $d$ and $u$ are in the same left cell. It is known that for any distinguished involution $d^{\prime}$, the degree $h_{w, d^{\prime}, u}$ is less than the degree of $h_{u, d, u}$ if either $w \neq u$ or $d^{\prime} \neq d$ (see [L2, Theorems 1.8 and 1.10]). Thus the degree of $a_{w} h_{w, d^{\prime}, u}$ is less than the degree of $a_{u} h_{u, d, u}$ if either $w \neq u$ or $d^{\prime} \neq d$. Hence $\varphi_{c^{\prime}}\left(h_{c}^{\prime}\right)$ is nonzero.

Clearly, there are only finitely many $q$ such that $\varphi_{c^{\prime}, q}\left(h_{c^{\prime}}\right)$ is zero after specializing $v$ to a square root of $q$. According to [BO, Theorem 4], the ring $\mathbf{J}_{c^{\prime}}$ is semisimple, that is, its Jacobson radical is zero. So we can find a nonzero $q$ in $\mathbb{C}$ of infinite order and a simple $\mathbf{J}_{c^{\prime}}$-module $E^{\prime}$ such that $\varphi_{c^{\prime}, q}(h)=\varphi_{c^{\prime}, q}\left(h_{c^{\prime}}\right)$ is nonzero and its action on $E^{\prime}$ is nonzero.

According to [L4, Theorems 4.2 and 4.8], $E_{q}^{\prime}$ is isomorphic to a standard module $M_{q}\left(s^{\prime}, n^{\prime}, \rho\right)$ with $n^{\prime}$ in the nilpotent orbit $\mathcal{C}^{\prime}$ corresponding to $c^{\prime}$. Since $c^{\prime} \mathbb{Z}_{L R} c, \mathcal{C}^{\prime}$ is not in the closure of $\mathcal{C}$ (see [B, Theorem 4(b)]), so by 2.2(a), the image $H_{\overline{\mathcal{C}}}$ of $\left(i_{\overline{\mathcal{C}}}\right)_{*}$ acts on $E_{q}^{\prime}$ by zero. This contradicts that the action of $\varphi_{c^{\prime}, q}(h)$ on $E^{\prime}$ is nonzero. Therefore $H_{\overline{\mathcal{C}}}$ is contained in the two-sided ideal $H^{\leq c}$.

2.5. In this subsection all tensor products are over $\mathbb{Z}$ except when other specifications are given.

Now we show that $H^{\leq c} \otimes \mathbb{Q}$ is equal to $H_{\overline{\mathcal{C}}} \otimes \mathbb{Q}$. If $\mathcal{C}$ is regular, then $\overline{\mathcal{C}}$ is the whole nilpotent cone and the corresponding two-sided cell $c$ contains the neutral element $e$; in this case, both $H_{\overline{\mathcal{C}}}$ and $H^{\leq c}$ are the whole Hecke algebra.

We use induction on the partial order $\leq_{L R}$ in the set of all two-sided cells of $W$. Assume that for all $c^{\prime}$ with $c \leq_{L R} c^{\prime}$ and $c^{\prime} \neq c$, we have $H_{\overline{\mathcal{C}}^{\prime}} \otimes \mathbb{Q}=H \leq c^{\prime} \otimes \mathbb{Q}$, where $\mathcal{C}^{\prime}$ is the nilpotent orbit corresponding to $c^{\prime}$.

We need to show $H_{\overline{\mathcal{C}}} \otimes \mathbb{Q}=H^{\leq c} \otimes \mathbb{Q}$. Let $c^{\prime}$ be a two-sided cell different from $c$ such that $c \leq_{L R} c^{\prime}$ but there is no two-sided cell $c^{\prime \prime}$ between $c$ and $c^{\prime}$, i.e. no $c^{\prime \prime}$ such that $c \leq_{L R} c^{\prime \prime} \leq_{L R} c^{\prime}$ and $c \neq c^{\prime \prime} \neq c^{\prime}$.

Let $\mathbb{F}$ be an algebraic closure of $\mathbb{C}(v)$. We first show that $\mathbb{F} \otimes_{\mathcal{A}} H_{\overline{\mathcal{C}}}=\mathbb{F} \otimes_{\mathcal{A}} H^{\leq c}$. Assume this were not true. Note that $\mathbb{F}$ is isomorphic to $\mathbb{C}$ (noncanonically), so we can apply the results in [KL2]. By 2.4 and induction hypothesis, there would exist $w \in c$ such that $C_{w}$ is contained in $\mathbb{F} \otimes_{\mathcal{A}} H_{\overline{\mathcal{C}}^{\prime}}$ but not in $\mathbb{F} \otimes_{\mathcal{A}} H_{\overline{\mathcal{C}}}$. 
We claim that $C_{w}$ is not contained in $\mathbb{F} \otimes_{\mathcal{A}} H_{\overline{\mathcal{C}}^{\prime}-\mathcal{C}^{\prime}}$. Let $\mathcal{C}_{i}(i=1, \ldots, k)$ be nilpotent classes such that $\overline{\mathcal{C}}^{\prime}-\mathcal{C}^{\prime}$ is the union of $\overline{\mathcal{C}}_{1}, \ldots, \overline{\mathcal{C}}_{k}$ and $\overline{\mathcal{C}}_{i} \nsubseteq \overline{\mathcal{C}}_{j}$ whenever $1 \leq i \neq j \leq k$. By the choice of $\mathcal{C}^{\prime}$, we have $\mathcal{C}=\mathcal{C}_{i}$ for some $i$. It is no harm to assume that $\mathcal{C}=\mathcal{C}_{1}$. It is known that $\mathbb{F} \otimes_{\mathcal{A}} H_{\overline{\mathcal{C}}^{\prime}-\mathcal{C}^{\prime}}$ is the sum of all $\mathbb{F} \otimes_{\mathcal{A}} H_{\overline{\mathcal{C}}_{i}}, 1 \leq i \leq k$ (see $[\mathrm{KL} 2,5.3(\mathrm{e})]$ ). Since $\overline{\mathcal{C}} \nsubseteq \overline{\mathcal{C}}_{i}$ for $i \neq 1$, by [B, Theorem 4(b)] we know that $C_{w}$ is not in $H^{\leq c_{i}}$, where $c_{i}$ is the two-sided cell corresponding to $\mathcal{C}_{i}$. By 2.4 we see that $\mathbb{F} \otimes_{\mathcal{A}} H_{\overline{\mathcal{C}}_{i}}(i \geq 1)$ does not contain $C_{w}$. Assume that $C_{w}$ were contained in $\mathbb{F} \otimes_{\mathcal{A}} H_{\overline{\mathcal{C}}^{\prime}-\mathcal{C}^{\prime}}$. Then there would exist a subset $J$ of $\{1, \ldots, k\}$ and $h_{i} \in \mathbb{F} \otimes_{\mathcal{A}} H_{\overline{\mathcal{C}}_{i}}(i \in J)$ such that $C_{w}=\sum_{i \in J} h_{i}$ and $h_{i} \notin H_{\overline{\mathcal{C}}_{i^{\prime}}}$ for different $i, i^{\prime}$ in $J$. We may choose such a $J$ so that $\sum_{i \in J} i$ is minimal possible. Let $j$ be the largest number in $J$. Then $j>1$ since $C_{w}$ is not contained in $\mathbb{F} \otimes_{\mathcal{A}} H_{\overline{\mathcal{C}}_{1}}$ (recall that $\mathcal{C}_{1}=\mathcal{C}$ ).

Let $C_{j}^{\prime}$ be a nilpotent class in $\overline{\mathcal{C}}_{j}$ such that $h_{j}$ is in $\mathbb{F} \otimes_{\mathcal{A}} H_{\overline{\mathcal{C}}_{j}^{\prime}}$ but not in $\mathbb{F} \otimes_{\mathcal{A}} H_{\overline{\mathcal{C}}_{j}^{\prime}-\mathcal{C}_{j}^{\prime}}$. Thus the image in $M_{\mathcal{C}_{j}^{\prime}}=\mathbb{F} \otimes_{\mathcal{A}} K^{G \times \mathbb{C}^{*}}\left(Z_{\mathcal{C}_{j}^{\prime}}\right)=\mathbb{F} \otimes_{\mathcal{A}} H_{\overline{\mathcal{C}}^{\prime}} / \mathbb{F} \otimes_{\mathcal{A}} H_{\overline{\mathcal{C}}_{j}^{\prime}-\mathcal{C}_{j}^{\prime}}$ of $h_{j}$ is nonzero. According to [KL2, Corollary 5.9], the action of each nonzero element in $\mathbb{F} \otimes_{\mathcal{A}}$ $H_{\overline{\mathcal{C}}_{j}^{\prime}} \backslash \mathbb{F} \otimes_{\mathcal{A}} H_{\overline{\mathcal{C}}_{j}^{\prime}-\mathcal{C}_{j}^{\prime}}$ on $M_{\mathcal{C}^{\prime}}$ is nonzero. The argument for [KL2, Proposition 5.13] implies that each nonzero element in $M_{\mathcal{C}_{j}^{\prime}}$ would have nonzero image in some standard quotient module of $M_{\mathcal{C}_{j}^{\prime}}$. Thus the action of $h_{j}$ on some standard quotient module $M_{v^{2}}\left(s, n_{j}^{\prime}, \rho^{\prime}\right)$ of $M_{\mathcal{C}_{j}^{\prime}}$ is nonzero, where $n_{j}^{\prime} \in \mathcal{C}_{j}^{\prime}$. Note that $\overline{\mathcal{C}}_{j}^{\prime} \nsubseteq \overline{\mathcal{C}}_{i}$ for any $i \in J$ with $i \neq j$ since $h_{j}$ is not in $\mathbb{F} \otimes_{\mathcal{A}} H_{\overline{\mathcal{C}}_{i}}$ if $i \neq j$. By 2.2(a), $h_{i}$ acts on $M_{v^{2}}\left(s, n_{j}^{\prime}, \rho^{\prime}\right)$ by zero if $i \neq j$. So $C_{w} M_{v^{2}}\left(s, n_{j}^{\prime}, \rho^{\prime}\right)=h_{j} M_{v^{2}}\left(s, n_{j}^{\prime}, \rho^{\prime}\right) \neq 0$. By 2.3(a), we get $\overline{\mathcal{C}}_{j}^{\prime} \subseteq \overline{\mathcal{C}}=\mathcal{C}_{1}$. This contradicts that $\sum_{i \in J} i$ is minimal and $j>1$. Therefore $C_{w}$ is not contained in $\mathbb{F} \otimes_{\mathcal{A}}$ $H_{\overline{\mathcal{C}^{\prime}}-\mathcal{C}^{\prime}}$

Thus the image in $M_{\mathcal{C}^{\prime}}=\mathbb{F} \otimes_{\mathcal{A}} K^{G \times \mathbb{C}^{*}}\left(Z_{\mathcal{C}^{\prime}}\right)=\mathbb{F} \otimes_{\mathcal{A}} H_{\overline{\mathcal{C}}^{\prime}} / \mathbb{F} \otimes_{\mathcal{A}} H_{\overline{\mathcal{C}}^{\prime}-\mathcal{C}^{\prime}}$ of $C_{w}$ is nonzero. According to [KL2, Corollary 5.9], the action of each nonzero element in $\mathbb{F} \otimes_{\mathcal{A}}$ $H_{\overline{\mathcal{C}}^{\prime}} \backslash \mathbb{F} \otimes_{\mathcal{A}} H_{\overline{\mathcal{C}}^{\prime}-\mathcal{C}^{\prime}}$ on $M_{\mathcal{C}^{\prime}}$ is nonzero. The argument for [KL2, Proposition 5.13] implies that each nonzero element in $M_{\mathcal{C}^{\prime}}$ would have nonzero image in some standard quotient module of $M_{\mathcal{C}^{\prime}}$. Thus the action of $C_{w}$ on some standard quotient module $M_{v^{2}}\left(s, n^{\prime}, \rho\right)$ of $M_{\mathcal{C}^{\prime}}$ is nonzero, where $n^{\prime} \in \mathcal{C}^{\prime}$. According to 2.3(a), we have $\overline{\mathcal{C}}^{\prime} \subseteq \overline{\mathcal{C}}$. By Theorem 4(b) in $[\mathrm{B}]$, we get $c^{\prime} \leq_{L R} \quad c$. This contradicts our assumption $c^{\prime} \neq c \leq_{L R} c^{\prime}$. So we have $\mathbb{F} \otimes_{\mathcal{A}} H_{\overline{\mathcal{C}}}=\mathbb{F} \otimes_{\mathcal{A}} H^{\leq c}$.

Thus for each $w \in c$, we can find a nonzero $a \in \mathbb{F}$ such that $a C_{w}$ is in $H_{\overline{\mathcal{C}}}$. Clearly, we must have $a \in \mathcal{A}$. Now we show that $\mathbf{K}^{G \times \mathbb{C}^{*}}\left(Z_{Y}\right)$ is a free $\mathbb{C}\left[v, v^{-1}\right]$-module for any $G$-stable locally closed subvariety $Y$ of $\mathcal{N}$. According to [KL2, 5.3] we may assume that $Y$ is a nilpotent orbit $\mathcal{C}$. It is enough to show that the completion of $\mathbf{K}^{G \times \mathbb{C}^{*}}\left(Z_{\mathcal{C}}\right)$ at any semisimple class in $G \times \mathbb{C}^{*}$ is free over $\mathbb{C}\left[v, v^{-1}\right]$. Using [KL2, 5.6] it is enough to show that the right hand side of 5.6(a) in [KL2] is free. This follows from [KL2, (13)]; the assumption there is satisfied by [KL2, 4.1]. Using [KL2, 5.3] we know that as a free $\mathbb{C}\left[v, v^{-1}\right]$-module, $H_{\overline{\mathcal{C}^{\prime}}} \otimes \mathbb{C}$ is a direct sum of $H_{\overline{\mathcal{C}}} \otimes \mathbb{C}$ and $\mathbf{K}^{G \times \mathbb{C}^{*}}\left(Z_{\overline{\mathcal{C}}^{\prime}-\overline{\mathcal{C}}}\right)$. By assumption, $H_{\overline{\mathcal{C}}^{\prime}} \otimes \mathbb{Q}=H^{\leq c^{\prime}} \otimes \mathbb{Q}$, thus $H_{\overline{\mathcal{C}}^{\prime}} \otimes \mathbb{Q}$ is a free $\mathbb{Q}\left[v, v^{-1}\right]$-module and contains $C_{w}$. These imply that if $a C_{w} \in H_{\overline{\mathcal{C}}}$ for some nonzero $a \in \mathcal{A}$ then $C_{w} \in H_{\overline{\mathcal{C}}} \otimes \mathbb{C}$. Therefore we 
can find a nonzero complex number $a$ such that $a C_{w}$ is in $H_{\overline{\mathcal{C}}}$. Obviously $a \in \mathbb{Z}$. Thus $H \leq c \otimes \mathbb{Q}$ is contained in $H_{\overline{\mathcal{C}}} \otimes \mathbb{Q}$. By 2.4 we then have $H^{\leq c} \otimes \mathbb{Q}=H_{\overline{\mathcal{C}}} \otimes \mathbb{Q}$. Theorem 1.5 is proved.

\section{An approach based on Theorem 4(a) in [B]}

In this section we discuss a nice possible approach to the main result of the present paper based on Theorem 4(a) in $[\mathrm{B}]$; this was suggested by the referee. Let $\Gamma$ be the union of all canonical left cells of $W$, and $I$ the left ideal of $H$ generated by all $C_{w}, w \notin \Gamma$. Then $M=H / I$ is the anti-spherical module. Moreover, the images in $M$ of all $C_{w}, w \in \Gamma$, form a basis of $M$. For each two-sided cell $c$ of $W$, let $M_{\leq c}$ be the submodule of $M$ spanned by the images of all $C_{w}, w \in \Gamma$ and $w \leq_{L R} c$.

According to Arkhipov and Bezrukavnikov (see Subsection 1.1.2 in [AB]), as an $H$ module, $M$ is isomorphic to $K^{G \times \mathbb{C}^{*}}(\Lambda)$ (see Subsection 10.1 in [L6] for the definition of the $H$-module structure on $K^{G \times \mathbb{C}^{*}}(\Lambda)$ ). Let $\mathcal{C}$ be the nilpotent class in $\mathcal{N}$ corresponding to the two-sided cell $c$ under Lusztig's bijection. Let $\Lambda_{\overline{\mathcal{C}}}=\{(N, \mathfrak{b}) \in \Lambda$ । $N \in \overline{\mathcal{C}}\}$. Then the inclusion $j_{\overline{\mathcal{C}}}: \Lambda_{\overline{\mathcal{C}}} \rightarrow \Lambda$ induces an $H$-module homomorphism $\left(j_{\overline{\mathcal{C}}}\right)_{*}: K^{G \times \mathbb{C}^{*}}\left(\Lambda_{\overline{\mathcal{C}}}\right) \rightarrow K^{G \times \mathbb{C}^{*}}(\Lambda)$. A variation of Theorem 4(a) in [B] implies that the image $\operatorname{Im}\left(j_{\overline{\mathcal{C}}}\right)_{*}$ of $\left(j_{\overline{\mathcal{C}}}\right)_{*}$ is $M_{\leq c}$ if we identify $M$ with $K^{G \times \mathbb{C}^{*}}(\Lambda)$.

Since each left cell in a two-sided cell has a nonempty intersection with any right cell in the same two-sided cell, we see that for a two-sided cell $c$, the two-sided ideal $H \leq c$ of $H$ spanned by all $C_{w}\left(w \leq_{L R} c\right)$ is the annihilator of $M / M_{\leq c}$.

Let $\mathcal{C}$ be the nilpotent class corresponding to the two-sided cell $c$. Then naturally one hopes to prove that the image $\operatorname{Im}\left(i_{\overline{\mathcal{C}}}\right)_{*}$ of the map $\left(i_{\overline{\mathcal{C}}}\right)_{*}: K^{G \times \mathbb{C}^{*}}\left(Z_{\overline{\mathcal{C}}}\right) \rightarrow K^{G \times \mathbb{C}^{*}}(Z)=H$ coincides with the two-sided ideal $H^{\leq c}$ by using the above characterizations for $M_{\leq c}$ and $H^{\leq c}$. A natural way to reach this coincidence is to prove the following two statements:

(a) $K^{G \times \mathbb{C}^{*}}\left(\Lambda \backslash \Lambda_{\overline{\mathcal{C}}}\right)$ is isomorphic to $K^{G \times \mathbb{C}^{*}}(\Lambda) / \operatorname{Im}\left(j_{\overline{\mathcal{C}}}\right)_{*}$.

(b) If $x \in K^{G \times \mathbb{C}^{*}}(Z)$ annihilates $K^{G \times \mathbb{C}^{*}}(\Lambda) / \operatorname{Im}\left(j_{\overline{\mathcal{C}}}\right)_{*}$, then $x \in \operatorname{Im}\left(i_{\overline{\mathcal{C}}}\right)_{*}$.

(a) implies that the image $\operatorname{Im}\left(i_{\overline{\mathcal{C}}}\right)_{*}$ is in $H^{\leq c}$, and (b) implies that this image contains $H^{\leq c}$. Unfortunately, the author has not been able to prove these two statements. See comments in Subsection 4.2 for some ideas.

\section{Some comments}

4.1. If one can show that $K^{G \times \mathbb{C}^{*}}\left(Z_{\mathcal{C}}\right)$ is a free $\mathbb{Z}$-module for any nilpotent orbit $\mathcal{C}$, then the argument in 2.5 shows that the image of $\left(i_{\overline{\mathcal{C}}}\right)_{*}$ in $H=K^{G \times \mathbb{C}^{*}}(Z)$ contains $H^{\leq c}$, where $c$ is the two-sided cell corresponding to $\mathcal{C}$. Then Ginzburg's conjecture would be proved. In fact, it seems that one can expect more. More precisely, it is likely the following result is true. 
(a) $K^{G \times \mathbb{C}^{*}}\left(Z_{\mathcal{C}}\right)$ is a free $\mathcal{A}$-module and $K_{1}^{G \times \mathbb{C}^{*}}\left(Z_{\mathcal{C}}\right)=0$ for all nilpotent orbits $\mathcal{C}$. (We refer to [CG, Section 5.2] and [Q] for the definition of the functor $K_{i}^{G}$.)

If (a) is true, then we also have

(b) The map $\left(i_{\overline{\mathcal{C}}}\right)_{*}: K^{G \times \mathbb{C}^{*}}\left(Z_{\overline{\mathcal{C}}}\right) \rightarrow K^{G \times \mathbb{C}^{*}}(Z)$ is injective.

We explain some evidence for (a) and prove it for $G=G L_{n}(\mathbb{C}), \operatorname{Sp}_{4}(\mathbb{C})$ and type $G_{2}$. Let $N$ be a nilpotent element in $\mathcal{C}$, and $\mathcal{B}_{N}$ be the variety of Borel subalgebras of $\mathfrak{g}$ containing $N$. By the Jacobson-Morozov theorem, there exists a homomorphism $\varphi: S L_{2}(\mathbb{C}) \rightarrow G$ such that $d \varphi\left(\begin{array}{ll}0 & 1 \\ 0 & 0\end{array}\right)=N$. For $z$ in $\mathbb{C}^{*}$, let $d_{z}=\left(\begin{array}{cc}z & 0 \\ 0 & z^{-1}\end{array}\right)$. Following Kazhdan and Lusztig [KL2, 2.4], we define $Q_{N}=\left\{(g, z) \in G \times \mathbb{C}^{*} \mid \operatorname{ad}(g) N=z^{2} N\right\}$. Then $Q_{N}$ is a closed subgroup of $G \times \mathbb{C}^{*}$. Let $x=(g, z) \in Q_{N}$ act on $\left(G \times \mathbb{C}^{*}\right) \times \mathcal{B}_{N} \times \mathcal{B}_{N}$ by $x\left(y, \mathfrak{b}, \mathfrak{b}^{\prime}\right)=\left(y x^{-1}, \operatorname{ad}(g) \mathfrak{b}, \operatorname{ad}(g) \mathfrak{b}^{\prime}\right)$. Then $Z_{\mathcal{C}}$ is isomorphic to the quotient space $Q_{N} \backslash\left(\left(G \times \mathbb{C}^{*}\right) \times \mathcal{B}_{N} \times \mathcal{B}_{N}\right)$. Thus we have $K_{i}^{G \times \mathbb{C}^{*}}\left(Z_{\mathcal{C}}\right)=K_{i}^{Q_{N}}\left(\mathcal{B}_{N} \times \mathcal{B}_{N}\right)($ see [KL2] 5.5] and [Th1, Prop. 6.2]). It is known that $Q_{\varphi}=\left\{(g, z) \in G \times \mathbb{C}^{*} \mid g \varphi(x) g^{-1}=\right.$ $\varphi\left(d_{z} x d_{z}^{-1}\right)$ for all $\left.x \in S L_{2}(\mathbb{C})\right\}$ is a maximal reductive subgroup of $Q_{N}$ (see [KL2, 2.4(d)]). So we have $K_{i}^{Q_{N}}\left(\mathcal{B}_{N} \times \mathcal{B}_{N}\right)=K_{i}^{Q_{\varphi}}\left(\mathcal{B}_{N} \times \mathcal{B}_{N}\right)$ (see [CG, 5.2.18]).

Let $P$ be the parabolic subgroup of $G$ associated to $N$ (see [DLP, 1.12]). Then we know that the intersection $\mathcal{B}_{N, \mathscr{O}}$ of $\mathcal{B}_{N}$ with any $P$-orbit $\mathscr{O}$ on $\mathcal{B}$ is smooth. The torus $\mathcal{D}=\left\{\varphi\left(d_{z}\right) \mid z \in \mathbb{C}^{*}\right\}$ is a subgroup of $P$ and acts on $\mathcal{B}_{N, \mathscr{O}}$, and $\mathcal{B}_{N, \mathscr{O}}$ is a vector bundle over the $\mathcal{D}$-fixed point set $\mathcal{B}_{N, \mathscr{O}}^{\mathcal{D}}$ (see [DLP, 3.4(d)]). Since the action of $Q_{\varphi}$ on $\mathcal{B}_{N, \mathscr{O}}$ commutes with the action of $\mathcal{D}$, according to $[\mathrm{BB}]$, this vector bundle is isomorphic to a $Q_{\varphi}$-stable subbundle of $\left.T\left(\mathcal{B}_{N, \mathscr{O}}\right)\right|_{\mathcal{B}_{N, \mathscr{O}}^{\mathcal{D}}}$, where $T\left(\mathcal{B}_{N, \mathscr{O}}\right)$ is the tangent bundle of $\mathcal{B}_{N, \mathscr{O}}$. Thus the vector bundle is $Q_{\varphi}$-equivariant, so that the computation of $K_{i}^{Q_{\varphi}}\left(\mathcal{B}_{N} \times \mathcal{B}_{N}\right)$ is reduced to the computation of $K_{i}^{Q_{\varphi}}\left(\mathcal{B}_{N, \mathscr{O}}^{\mathcal{D}} \times \mathcal{B}_{N, \mathscr{O}^{\prime}}^{\mathcal{D}}\right)$ for various $P$-orbits $\mathscr{O}, \mathscr{O}^{\prime}$ on $\mathcal{B}$ (see Theorems 2.7 and 4.1 in [Th1], or Theorems 5.4.17 and 5.2.14 in [CG]). Note that $C_{\varphi}=\left\{g \varphi\left(d_{z}^{-1}\right) \mid(g, z) \in Q_{\varphi}\right\}$ is a maximal reductive subgroup of the centralizer $C_{G}(N)$ of $N$ (see [BV, 2.4]) and the map $(g, z) \mapsto\left(g \varphi\left(d_{z}^{-1}\right), z\right)$ defines an isomorphism from $Q_{\varphi}$ to $C_{\varphi} \times \mathbb{C}^{*}$. Thus we have $K_{i}^{Q_{\varphi}}\left(\mathcal{B}_{N, \mathscr{O}}^{\mathcal{D}} \times \mathcal{B}_{N, \mathscr{O}^{\prime}}^{\mathcal{D}}\right)=K_{i}^{C_{\varphi} \times \mathbb{C}^{*}}\left(\mathcal{B}_{N, \mathscr{O}}^{\mathcal{D}} \times \mathcal{B}_{N, \mathscr{O}^{\prime}}^{\mathcal{D}}\right)$. Now the factor $\mathbb{C}^{*}$ and the group $\mathcal{D}$ act on $\mathcal{B}_{N, \mathscr{O}}^{\mathcal{D}} \times \mathcal{B}_{N, \mathscr{O}^{\prime}}^{\mathcal{D}}$ trivially, we therefore have $K_{i}^{Q_{\varphi}}\left(\mathcal{B}_{N, \mathscr{O}}^{\mathcal{D}} \times \mathcal{B}_{N, \mathscr{O}^{\prime}}^{\mathcal{D}}\right)=K_{i}^{C_{\varphi}}\left(\mathcal{B}_{N, \mathscr{O}}^{\mathcal{D}} \times \mathcal{B}_{N, \mathscr{O}^{\prime}}^{\mathcal{D}}\right) \otimes R_{\mathbb{C} *}$ (see [CG (5.2.4)], the argument there works for higher $K$-groups). Note that we have identified $R_{\mathbb{C} *}$ with $\mathcal{A}=\mathbb{Z}\left[v, v^{-1}\right]$. Thus the statement (a) is equivalent to the following one.

(c) $K_{i}^{C_{\varphi}}\left(\mathcal{B}_{N, \mathscr{O}}^{\mathcal{D}} \times \mathcal{B}_{N, \mathscr{O}^{\prime}}^{\mathcal{D}}\right)$ is a free $\mathbb{Z}$-module for $i=0$ and is 0 for $i=1$.

The statement (c) seems much easier to access. The variety $\mathcal{B}_{N, \mathscr{O}}^{\mathcal{D}}$ and its fixed point set $\mathcal{B}_{N, \mathscr{O}}^{s, \mathcal{D}}$ for any semisimple element $s$ in $C_{\varphi}$ are smooth and have good homology properties. See [DLP].

4.2. Replacing $Z$ by $\Lambda$, we can state the counterparts of $4.1(a), 4.1(b)$ and $4.1(c)$ as follows. 
(a) $K^{G \times \mathbb{C}^{*}}\left(\Lambda_{\mathcal{C}}\right)$ is a free $\mathcal{A}$-module and $K_{1}^{G \times \mathbb{C}^{*}}\left(\Lambda_{\mathcal{C}}\right)=0$ for all nilpotent orbits $\mathcal{C}$.

If (a) is true, then we have

(b) The map $\left(i_{\overline{\mathcal{C}}}\right)_{*}: K^{G \times \mathbb{C}^{*}}\left(\Lambda_{\overline{\mathcal{C}}}\right) \rightarrow K^{G \times \mathbb{C}^{*}}(\Lambda)$ is injective.

As in 4.1 , the statement (a) is equivalent to the following one:

(c) $K_{i}^{C_{\varphi}}\left(\mathcal{B}_{N, \mathscr{O}}^{\mathcal{D}}\right)$ is a free $\mathbb{Z}$-module for $i=0$ and is 0 for $i=1$.

It is easy to check that the statement (a) implies 3(a). Also (a) is helpful to understand the statement 3(b).

Proposition 4.3. The statements 4.1(a) and 4.2(a) are true for $G L_{n}(\mathbb{C}), S p_{4}(\mathbb{C})$ and type $G_{2}$. In particular, Ginzburg's conjecture is true in these cases.

Proof. We only need to prove statements 4.1(c) and 4.2(c). For $G=G L_{n}(\mathbb{C})$, we know that $\mathcal{B}_{N, \mathscr{O}}^{\mathcal{D}}$ has an $\alpha$-partition into subsets which are affine space bundles over the flag variety $\mathcal{B}^{\prime}$ of $C_{\varphi}$ (see Theorems 2.2 and 2.4(a) in [X2]). In this case, 4.1(a) and 4.2(a) are true since we are reduced to computing $K_{i}^{C_{\varphi}}\left(\mathcal{B}^{\prime} \times \mathcal{B}^{\prime}\right)$ and $K_{i}^{C_{\varphi}}\left(\mathcal{B}^{\prime}\right)$ (cf. [CG, Lemma 5.5.1] and the argument for [L7, Lemma 1.6]). For $G=S p_{4}(\mathbb{C})$ or type $G_{2}$, we know that $\mathcal{B}_{N, \mathscr{O}}^{\mathcal{D}}$ is either empty or the flag variety of $C_{\varphi}$ if $N$ is not subregular (see Prop. 4.2(i) and Section 4.4 in [X2]). In this case, we are also reduced to computing $K_{i}^{C_{\varphi}}\left(\mathcal{B}^{\prime} \times \mathcal{B}^{\prime}\right)$ and $K_{i}^{C_{\varphi}}\left(\mathcal{B}^{\prime}\right)$ (loc.cit.), so 4.1(a) and 4.2(a) are true. If $N$ is subregular, then $\mathcal{B}_{N}$ is a Dynkin curve and it is easy to see that $\mathcal{B}_{N, \mathscr{O}}^{\mathcal{D}}$ is either a projective line or a finite set (see Prop. 4.2(ii) and Section 4.4 in [X2] for a computable description of $\mathcal{B}_{N}$ ). The computation for $K_{i}^{C_{\varphi}}\left(\mathcal{B}_{N, \mathscr{O}}^{\mathcal{D}} \times \mathcal{B}_{N, \mathscr{O}^{\prime}}^{\mathcal{D}}\right)$ and $K_{i}^{C_{\varphi}}\left(\mathcal{B}_{N, \mathscr{O}}^{\mathcal{D}}\right)$ is easy, they are free $\mathbb{Z}$-modules for $i=0$ (see 4.3 (b) and 4.4 in [ [X2]), and are 0 for $i=1$ (since this is true for a projective line and a finite set). The proposition is proved.

Remark. For $G L_{n}(\mathbb{C})$, this proposition also provides another proof for the main result of [TX], where results of [T1] are used.

Proposition 4.4. Assume that $C_{\varphi}$ is connected. Then

(a) $K^{C_{\varphi}}\left(\mathcal{B}_{N} \times \mathcal{B}_{N}\right)$ is a free $\mathbb{Z}$-module.

(b) $K^{Q_{\varphi}}\left(\mathcal{B}_{N} \times \mathcal{B}_{N}\right)$ is a free $\mathcal{A}$-module. That is, $K^{G \times \mathbb{C} *}\left(Z_{G . N}\right)$ is a free $\mathcal{A}$-module.

Proof. Let $T$ be a maximal torus of $C_{\varphi}$. According to [Th2, (1.11)], we have a split monomorphism $K^{C_{\varphi}}\left(\mathcal{B}_{N} \times \mathcal{B}_{N}\right) \rightarrow K^{T}\left(\mathcal{B}_{N} \times \mathcal{B}_{N}\right)$. Similar to the argument for [L7] Lemma 1.13(d)], we see that $K^{T}\left(\mathcal{B}_{N} \times \mathcal{B}_{N}\right)$ is a free $R_{T}$-module. (a) follows.

The reasoning for (b) is similar since $Q_{\varphi}$ is isomorphic to $C_{\varphi} \times \mathbb{C}^{*}$ and the monomorphism $K^{Q_{\varphi}}\left(\mathcal{B}_{N} \times \mathcal{B}_{N}\right) \rightarrow K^{T \times \mathbb{C}^{*}}\left(\mathcal{B}_{N} \times \mathcal{B}_{N}\right)$ is split. The proposition is proved.

Remark. If $G=G L_{n}(\mathbb{C})$, then all $C_{\varphi}$ are connected and have simply connected derived group. In this case $K^{Q_{\varphi}}\left(\mathcal{B}_{N} \times \mathcal{B}_{N}\right)$ is a free $R_{Q_{\varphi}}$-module since $R_{Q_{\varphi}}=R_{C_{\varphi}} \otimes \mathcal{A}$ and $R_{T \times \mathbb{C}^{*}}$ is a free $R_{C_{\varphi}} \otimes \mathcal{A}$-module. Combining this, Subsection 2.4 and the argument in Subsection 2.5 we obtain a different proof of the main result in [TX]. 
4.5. The $K$-groups $K^{F}\left(\mathcal{B}_{N}\right)$ and $K^{F}\left(\mathcal{B}_{N} \times \mathcal{B}_{N}\right)$ are important in representation theory of affine Hecke algebras for $F$ being $Q_{\varphi}, C_{\varphi}$ or a torus of $Q_{\varphi}$ (see [KL2, L7]). For the nilpotent element $N$, in [L4, 10.5] Lusztig conjectured that there exists a finite $C_{\varphi}$-set $Y$ which plays a key role in understanding the based ring of the two-sided cell corresponding to G.N. It seems that as $R_{C_{\varphi}}$-modules, $K^{C_{\varphi}}(Y)$ and $K^{C_{\varphi}}(Y \times Y)$ are isomorphic to $K^{C_{\varphi}}\left(\mathcal{B}_{N}\right)$ and $K^{C_{\varphi}}\left(\mathcal{B}_{N} \times \mathcal{B}_{N}\right)$ respectively. Let $X=\mathcal{B}_{N}$ or $\mathcal{B}_{N} \times \mathcal{B}_{N}$. In view of [L4] 10.5] one may hope to find a canonical $\mathbb{Z}$-basis of $K^{C_{\varphi}}(X)$ and a canonical $\mathcal{A}$-basis of $K^{Q_{\varphi}}(X)$ in the spirit of [L6, L7]. Moreover, there should exist a natural bijection between the elements of the canonical basis of $K^{F}\left(\mathcal{B}_{N} \times \mathcal{B}_{N}\right)\left(F=C_{\varphi}\right.$ or $\left.Q_{\varphi}\right)$ and the elements of the two-sided cell corresponding to G.N.

Acknowledgments. I thank Professor G. Lusztig for very helpful correspondence and for providing the argument for the freeness of $\mathbf{K}^{G \times \mathbb{C}^{*}}\left(Z_{\mathcal{C}}\right)$ over $\mathbb{C}\left[v, v^{-1}\right]$. I am grateful to Professor T. Tanisaki for helpful correspondence and to Professor Jianzhong Pan for a helpful conversation. I am indebted to the referee for very helpful comments and an insightful suggestion.

This research was partially supported by Natural Sciences Foundation of China (No. 10671193).

\section{References}

[AB] Arkhipov, S., Bezrukavnikov, R.: Perverse sheaves on affine flags and Langlands dual groups. Israel J. Math. 170, 135-183 (2009) Zbl pre05601711 MR 2506322

[BV] Barbasch, D., Vogan, D.: Unipotent representation of complex semisimple groups. Ann. of Math. 121, 41-110 (1985) Zbl 0582.22007 MR 0782556

[B] Bezrukavnikov, R.: Perverse sheaves on affine flags and nilpotent cone of the Langlands dual group. Israel J. Math. 170, 185-206 (2009) Zbl pre05601712 MR 2506323

[BO] Bezrukavnikov, R., Ostrik, V.: On tensor categories attached to cells in affine Weyl groups, II. In: Representation Theory of Algebraic Groups and Quantum Groups, Adv. Stud. Pure Math. 40, Math. Soc. Japan, Tokyo, 101-119 (2004) Zbl 1078.20045 MR 2074591

[BB] Białynicki-Birula, A.: Some theorems on actions of algebraic groups. Ann. of Math. 98, 480-497 (1973) Zbl 0275.14007 MR 0366940

[CG] Chriss, N., Ginzburg, V.: Representation Theory and Complex Geometry. Birkhäuser Boston, Boston, MA (1997) Zbl 0879.22001 MR 1433132

[DLP] De Concini, C., Lusztig, G., Procesi, C.: Homology of the zero-set of a nilpotent vector field on a flag manifold. J. Amer. Math. Soc. 1, 15-34 (1988) Zbl 0646.14034 MR 0924700

[G1] Ginzburg, V.: Lagrangian construction of representations of Hecke algebras. Adv. Math. 63, 100-112 (1987) MR 0871082

[G2] Ginzburg, V.: Geometrical aspects of representation theory. In: Proc. International Congress of Mathematicians, Vol. 1 (Berkeley, CA, 1986), Amer. Math. Soc., Providence, RI, 840848 (1987) Zbl 0667.20033 MR 0934285

[KL1] Kazhdan, D., Lusztig, G.: Representations of Coxeter groups and Hecke algebras. Invent. Math. 53, 165-184 (1979) Zbl 0499.20035 MR 0560412

[KL2] Kazhdan, D., Lusztig, G.: Proof of the Deligne-Langlands conjecture for Hecke algebras. Invent. Math. 87, 153-215 (1987) Zbl 0613.22004 MR 0862716

[L1] Lusztig, G.: Cells in affine Weyl groups. In: Algebraic Groups and Related Topics (Kyoto/ Nagoya, 1983), Adv. Stud. Pure Math. 6, Kinokuniya and North-Holland, 255-287 (1985) Zbl 0569.20032 MR 0803338 
[L2] Lusztig, G.: Cells in affine Weyl groups. II. J. Algebra 109, 536-548 (1987) Zbl 0625.20032 MR 0902967

[L3] Lusztig, G.: Cells in affine Weyl groups, III. J. Fac. Sci. Univ. Tokyo Sect. IA Math. 34, 223-243 (1987) Zbl 0631.20028 MR 0914020

[L4] Lusztig, G.: Cells in affine Weyl groups. IV. J. Fac. Sci. Univ. Tokyo Sect. IA Math. 36, 297-328 (1989) Zbl 0688.20020 MR 1015001

[L5] Lusztig, G.: Representations of affine Hecke algebras. Astérisque 171-172, 73-84 (1989) Zbl 0699.22027 MR 1021500

[L6] Lusztig, G.: Bases in equivariant $K$-theory. Represent. Theory 2, 298-369 (1998) Zbl 0901.20034 MR 1637973

[L7] Lusztig, G.: Bases in equivariant $K$-theory, II. Represent. Theory 3, 281-353 (1999) Zbl 0999.20036 MR 1714628

[Q] Quillen, D.: Higher algebraic K-theory I. In: Higher $K$-Theory, Lecture Notes in Math. 341, Springer, 85-147 (1972) Zbl 0292.18004 MR 0338129

[T1] Tanisaki, T.: Hodge modules, equivariant $K$-theory and Hecke algebras. Publ. Res. Inst. Math. Sci. 23, 841-879 (1987) Zbl 0655.14004 MR 0934674

[T2] Tanisaki, T.: Representations of semisimple Lie groups and $D$-modules. Sugaku Expositions 4, 43-61 (1991) Zbl 0695.22007 MR 1121306

[TX] Tanisaki, T., Xi, N.: Kazhdan-Lusztig basis and a geometric filtration of an affine Hecke algebra. Nagoya Math. J. 182, 285-311 (2006) Zbl 1165.20003 MR 2235345

[Th1] Thomason, R. W.: Algebraic K-theory of group scheme actions. In: Algebraic Topology and Algebraic K-Theory, Ann. of Math. Stud. 113, Princeton Univ. Press, 539-563 (1987) Zbl 0701.19002 MR 0921490

[Th2] Thomason, R. W.: Equivariant algebraic vs. topological K-homology Atiyah-Segal-style. Duke Math. J. 56, 589-636 (1988) Zbl 0655.55002 MR 0948534

[X1] Xi, N.: Representations of Affine Hecke Algebras. Lecture Notes in Math. 1587, Springer, Berlin (1994) Zbl 0817.20051 MR 1320509

[X2] Xi, N.: A partition of the Springer fibers $\mathcal{B}_{N}$ for type $A_{n-1}, B_{2}, G_{2}$ and some applications. Indag. Math. (N.S.) 10, 307-320 (1999) Zbl 1029.20022 MR 1816223

[X3] Xi, N.: Representations of affine Hecke algebras and based ring of affine Weyl groups. J. Amer. Math. Soc. 20, 211-217 (2007) Zbl 1150.20005 MR 2257401 